\title{
Revolute joints with clearance in multibody systems
}

\author{
P. Flores ${ }^{a}$, J. Ambrósio b,* \\ ${ }^{a}$ Departamento de Engenharia Mecânica, Universidade do Minho, Campus de Azurém, 4800-058 Guimarães, Portugal \\ ${ }^{\mathrm{b}}$ Instituto de Engenharia Mecânica (IDMEC), Instituto Superior Técnico, Av. Rovisco Pais, 1049-001 Lisboa, Portugal
}

Received 10 March 2004; accepted 12 March 2004

Available online 25 May 2004

\begin{abstract}
A computational methodology for dynamic analysis of multibody mechanical systems with joint clearance is presented in this work. Clearances always exist in real joints in order to ensure the correct relative motion between the connected bodies being the gap associated to them a result of machining tolerance, wear, and local deformations. Clearance at different joints is the source for impact forces, resulting in wear and tear of the joints, and consequently the degradation of the system performance. The model for planar revolute joints is based on a thorough geometric description of contact conditions and on a continuous contact force model, which represents the impact forces. It is shown that the model proposed here lead to realistic contact forces. These forces correlate well with the joint reaction forces of an ideal revolute joint, which correspond to a null joint clearance. The application to the analysis of a simple planar multibody system illustrates the use of the different models proposed.
\end{abstract}

(c) 2004 Elsevier Ltd. All rights reserved.

Keywords: Clearance joints; Multibody dynamics; Contact forces; Impact analysis

\section{Introduction}

In general dynamic analysis of multibody mechanical systems it is assumed that the kinematic joints are ideal or perfect, that is, clearance, local deformations, wear, and lubrication effects are neglected. However, in a real mechanical kinematical joint a gap is always present. Such clearance is necessary to allow the relative motion between the connected bodies and to permit the components assemblage as well. For instance, in a journalbearing joint there is a radial clearance allowing for the relative motion between the journal and the bearing. This clearance is inevitable due to the machining tolerances, wear, material deformations, and imperfections. The presence of such joint gaps leads to degradation of the performance of mechanical systems in virtue of the impact forces that take place. Not only these impact

\footnotetext{
${ }^{*}$ Corresponding author. Tel.: +351-21-8417680; fax: +35121-8417915.

E-mail address: jorge@dem.ist.utl.pt (J. Ambrósio).
}

forces dissipate energy but they are also a source for vibrations and noise.

The general purpose computational tools used for design and analysis of mechanical systems, such as ADAMS [1] or DADS [2], have a wide number of modeling features that require the description of rigid or flexible bodies for which geometry, mass, center of mass, moment of inertia, and other relevant properties are defined. These codes also provide a large library of kinematic joints that constrain relative degrees of freedom between connected bodies. The kinematic joints available in these commercial programs are represented as ideal joints, i.e., there are no clearances or deformations in them.

The subject of the representation of real joints draw the attention of a large number of researchers that produced several theoretical and experimental works devoted to the dynamic simulation of mechanical systems with joint clearances [3-8]. Some of these works focus on the planar systems in which only one kinematic joint is modeled as clearance joint [6,7]. Bengisu et al. [8] presented a study of a four-bar mechanism with multiple 
joints with clearances. Some researchers also include the influence of the flexibility of the bodies in the dynamic performance of multibody systems besides the existence of gaps in the joints [5,9]. Based on different clearance models, Claro and Fernandes [10] presented a qualitative study of the performance of a slider-crank mechanism with a nonperfect revolute clearance joint.

While an ideal revolute joint imposes permanent kinematic constraints to the systems, a revolute joint with clearance has to be dealt with a different approach, for instance using force constraints [6]. For planar systems the existence of a radial clearance in a revolute joint removes the two kinematic constraints associated with the ideal revolute joint, and, hence, two extra degrees of freedom are introduced. Thus, the journal can move freely inside the bearing boundaries. When the journal reaches the bearing wall an impact takes place and contact forces control the dynamics of the joint.

In general, there are three main modeling strategies for mechanical systems with revolute clearance joints, namely, the massless link approach [11-13], the springdamper approach $[3,4,14]$, and the momentum exchange approach $[6,15]$. In the massless link approach, the presence of clearance at a joint is modeled by adding a link of zero mass that has a constant length equal to the radial clearance, as shown in Fig. 1. The result is a mechanism that has an additional degree of freedom, when compared with the system that has the ideal joint. In the spring-damper approach, the clearance is modeled by introducing a spring-damper element, represented in Fig. 2, which simulates the surface elasticity. This model does not represent the physical nature of energy transfer during the impact process. Moreover, there is a real difficulty in quantifying the parameters of the spring and damper elements. In the third model, shown in Fig. 3, the journal-bearing elements are considered as two colliding bodies and the contact forces control the dynamics of the clearance joint.

The existence of impacts in the joint lead to the appearance of high level of contact forces during dynamic analysis. The difference in radius between the bearing and the journal, which defines the radial clearance size, is directly associated to the model of contact

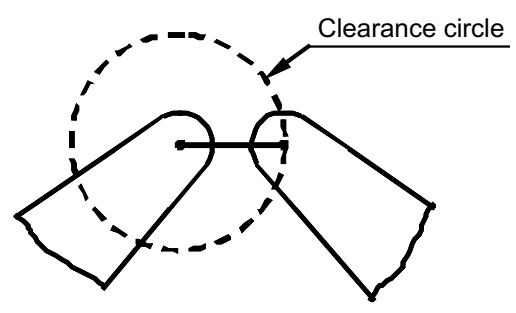

Fig. 1. Revolute clearance joint modeled by massless link approach.

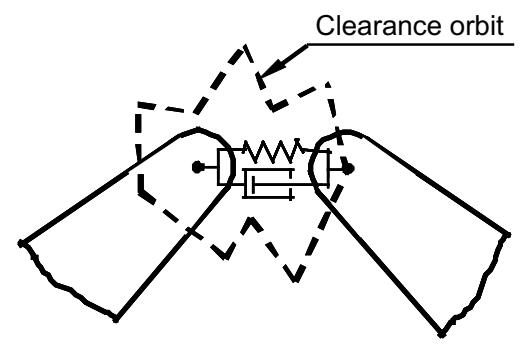

Fig. 2. Revolute clearance joint modeled by spring-damper approach.

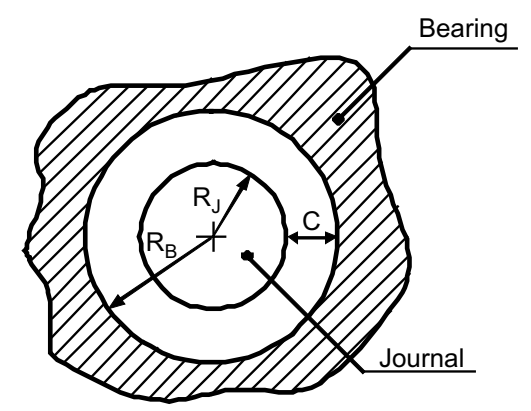

Fig. 3. Revolute clearance joint modeled as colliding bodies.

forces that develop during the motion of the system. In the first two models, the clearance is replaced by an equivalent component, which tries to simulate its behavior as closely as possible. The third model is more realistic, as it allows for the contact force models to develop as a function of the elasticity properties of contacting surfaces and it takes into account the dissipation of energy during the impact process.

The modeling of the impact in multibody systems is well described by two types of methods, namely continuous and discontinuous approaches [16,17]. Within the continuous approaches the methods commonly used are the continuous force model, which is in fact a penalty method, and the unilateral constraint methodology, based on complementary approaches [18]. The continuous contact force model represents the forces arising from the collisions, assuming that the force is a continuous function of deformation. In this model, when contact is detected, a force perpendicular to the plane of collision is applied. The contact force model can be linear, as in the Kelvin-Voigt model, or nonlinear, as represented by the Hertz law. For long impact durations this method is effective and accurate in so far as the instantaneous contact force is evaluated and introduced into the equations of motion of the system. In the second continuous approach, when contact is detected a kinematic constraint is introduced in the system equations. Such constraint is maintained while the reaction forces 
are compressive, and removed when the impacting bodies rebound from contact [19].

The second type of methods, the discontinuous model, assumes that the impact occurs instantaneously and that no change of the system configuration occurs during contact. The integration of equations of motion is halted at the time of impact and a momentum balance is performed to calculate the post impact velocities of the system components. The restitution coefficient is employed to quantify the dissipation energy in the process. This method is relatively efficient, however, the unknown duration of impact limits its application, mainly for long impact duration in which the system configuration changes significantly and the assumption of instantaneity of impact is no longer valid [20].

The main emphasis of this work is on the modeling revolute clearance joints in multibody mechanical systems. The contact between the journal and the bearing is modeled by using a continuous impact force model. The impact forces are then introduced into the system's equations of motion in order to analyze the dynamic behavior of the system.

In order to demonstrate the use of the methodology described throughout this work, an application to a planar slider-crank mechanism in which the revolute joint between the connecting rod and slider has a controlled clearance is presented.

\section{Equations of motion for multibody systems}

The position of a body reference frame is defined, in what follows, by a set of Cartesian coordinates. The position and orientation of rigid body $i$ is defined by

$\mathbf{q}_{i}^{*}=\left[\begin{array}{ll}\mathbf{r}_{i}^{\mathrm{T}} & \mathbf{p}_{i}^{\mathrm{T}}\end{array}\right]^{\mathrm{T}}$,

where $\mathbf{r}_{i}=\left[\begin{array}{lll}x & y & z\end{array}\right]^{\mathrm{T}}$ are the translation coordinates and $\mathbf{p}_{i}=\left[\begin{array}{llll}e_{0} & e_{1} & e_{2} & e_{3}\end{array}\right]^{\mathrm{T}}$ are the rotational coordinates, given here by Euler parameters. The velocities and accelerations of body $i$ use the angular velocities $\omega_{i}^{\prime}$ and accelerations $\dot{\omega}_{i}^{\prime}$ instead of the time derivatives of the Euler parameters. The velocities and accelerations of body $i$ are given by vectors

$\dot{\mathbf{q}}_{i}=\left[\begin{array}{ll}\dot{\mathbf{r}}_{i}^{\mathrm{T}} & \boldsymbol{\omega}_{i}^{\prime \mathrm{T}}\end{array}\right]^{\mathrm{T}}$

$\ddot{\mathbf{q}}_{i}=\left[\begin{array}{ll}\ddot{\mathbf{r}}_{i}^{\mathrm{T}} & \dot{\boldsymbol{\omega}}_{i}^{\prime \mathrm{T}}\end{array}\right]^{\mathrm{T}}$

In terms of the Cartesian coordinates, the equations of motion of an unconstrained multibody system are written as

$\mathbf{M} \ddot{\mathbf{q}}_{r}=\mathbf{g}$,

where $\mathbf{M}$ is a mass matrix and $\mathbf{g}$ is a force vector that contains the external and Coriolis forces acting on the bodies of the system. For a constrained multibody system the kinematical joints are described by a set of holonomic algebraic constraints

$\boldsymbol{\Phi}\left(\mathbf{q}_{r}, t\right)=\mathbf{0}$.

Using the Lagrange multipliers technique the constraints are added to the equations of motion. These are written together with the second time derivatives of the constraint equation (5). The set of equations that describe the motion of the multibody system is

$\left[\begin{array}{cc}\mathbf{M} & \boldsymbol{\Phi}_{q}^{\mathrm{T}} \\ \mathbf{\Phi}_{q} & \mathbf{0}\end{array}\right]\left[\begin{array}{c}\ddot{\mathbf{q}}_{r} \\ \boldsymbol{\lambda}\end{array}\right]=\left[\begin{array}{l}\mathbf{g} \\ \boldsymbol{\gamma}\end{array}\right]$,

where $\lambda$ is the vector of Lagrange multipliers and $\gamma$ is vector that groups all the terms of the acceleration constraint equations that depend on the velocities only, i.e.,

$\boldsymbol{\gamma}=-\left(\boldsymbol{\Phi}_{q} \dot{\mathbf{q}}\right)_{q} \dot{\mathbf{q}}_{r}-\boldsymbol{\Phi}_{t t}-2 \boldsymbol{\Phi}_{q t} \dot{\mathbf{q}}_{r}$

Eq. (6) is a differential-algebraic equation that has to be solved and the resulting accelerations integrated in time. However, because they do not use explicitly the position and velocity constraint equations there may be a drift in the system constraints. To avoid constraints violation during numerical integration, Baumgarte stabilization is used, and Eq. (6) modified as

$$
\left[\begin{array}{cc}
\mathbf{M} & \boldsymbol{\Phi}_{q}^{\mathrm{T}} \\
\mathbf{\Phi}_{q} & \mathbf{0}
\end{array}\right]\left[\begin{array}{c}
\ddot{\mathbf{q}}_{r} \\
\lambda
\end{array}\right]=\left[\begin{array}{c}
\mathbf{g} \\
\boldsymbol{\gamma}-2 \alpha \dot{\boldsymbol{\Phi}}-\beta^{2} \boldsymbol{\Phi}
\end{array}\right],
$$

where $\alpha$ and $\beta$ are positive constants that represent the feedback control parameters for the velocities and position constraint violations. The interested reader is referred to Nikravesh [21] and Baumgarte [22] for further details on the formulation used.

A set of initial conditions, positions and velocities, is required to start the dynamic simulation. The selection of the appropriate initial conditions plays a key role in the prediction of the dynamic performance of mechanical system. In the present work, the initial conditions are based on the results of kinematic simulation of mechanical system in which all the joints are assumed to be ideal. The use of numerical algorithms with automated adjust step size is particularly important in contact problems whose dynamic response is quite complex due to the sudden change in kinematic configuration. In such events, the use of a constant time step is computationally inefficient and the system could be overlooked due to insufficient time resolution. Thus, automated time step size adaptability is a crucial part of the dynamic solution procedure. Moreover, the abrupt configuration changes caused by rapid variation of contact forces results in stiff equations of motion for the system, since the natural frequency of the system are widely spread. Thus, the time step size must be adjusted in order to capture both the fast and low frequency components of the 
system response [21]. The integration process is performed here using a predictor-corrector algorithm with both variable step size and order [23].

\section{Kinematic aspects of revolute joints with clearance}

In standard multibody models it is assumed that the connecting points of two bodies linked by an ideal or perfect revolute joint are coincident. The introduction of the clearance in a revolute joint separates these two points as observed in Fig. 3. The difference in radius between the bearing and the journal, $c=R_{\mathrm{B}}-R_{\mathrm{J}}$, defines the size of radial clearance. Relative to the situation of an ideal joint, a revolute clearance joint introduces two extra degrees of freedom in the system, that is, the horizontal and vertical motion of the journal relative to the bearing center. In a noncontact situation, no constraints or forces are introduced by the journal-bearing. However, during the contact between the journal and bearing, impact forces develop. Thus, a revolute clearance joint does not constrain any degree of freedom from the mechanical system like the ideal joint, but imposes kinetic interactions that bound the journal to move within the bearing boundaries. Thus, whilst a perfect joint in a mechanical system imposes kinematic constraints, a revolute clearance joint deals with force constraints only. The dynamic behavior of the revolute clearance joint is treated as an eccentric impact between the journal and the bearing. When the contact takes place, an impact force law is applied and the resulting forces are introduced into the system's equations of motion.

Fig. 4 shows two bodies $i$ and $j$, the extremities of which define the bearing and journal, respectively. The center of mass of bodies $i$ and $j$ are $\mathrm{O}_{i}$ and $\mathrm{O}_{j}$, respectively. Body-fixed coordinates $\xi \eta$ are attached at the centers of mass, while $X Y$ coordinate frame represents

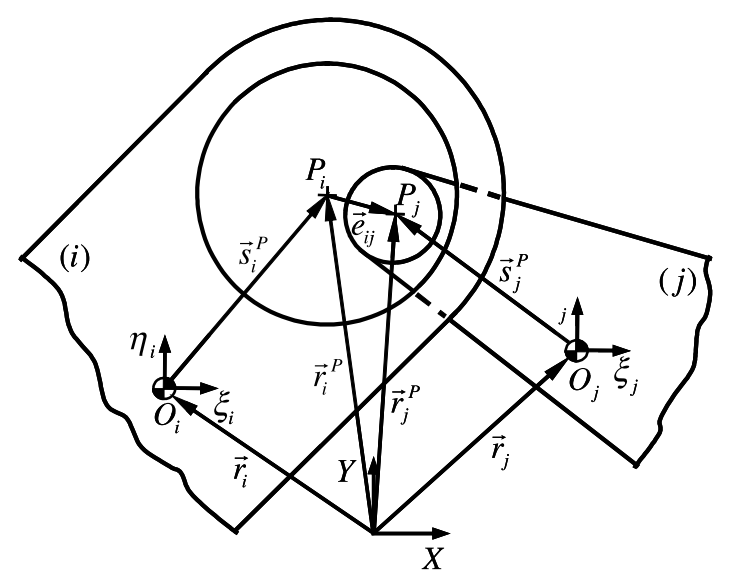

Fig. 4. General revolute clearance joint in a multibody system. the global coordinate system. Point $\mathrm{P}_{i}$ indicates the center of the bearing, and the center of the journal is defined at point $\mathrm{P}_{j}$. As displayed in Fig. 4, the eccentricity vector $\mathbf{e}_{i j}$, which connects the centers of the bearing and journal is given by

$\mathbf{e}_{i j}=\mathbf{r}_{j}^{\mathrm{P}}-\mathbf{r}_{i}^{\mathrm{P}}$,

where both $\mathbf{r}_{j}^{\mathrm{P}}$ and $\mathbf{r}_{i}^{\mathrm{P}}$ are described in global coordinates with respect to the inertial reference frame [21], that is,

$\mathbf{r}_{k}^{\mathrm{P}}=\mathbf{r}_{k}+\mathbf{A}_{k} \mathbf{s}_{k}^{\prime \mathrm{P}} \quad(k=i, j)$.

The magnitude of the eccentricity vector is evaluated as,

$e_{i j}=\sqrt{\mathbf{e}_{i j}^{\mathrm{T}} \mathbf{e}_{i j}}$.

A unit vector $\mathbf{n}$ normal to the plane of collision between the bearing and journal is defined by

$\mathbf{n}=\frac{\mathbf{e}_{i j}}{e_{i j}}$.

Note that the unit vector $\mathbf{n}$ has the same direction as the line of the centers of the bearing and journal.

With reference to Fig. 5, the penetration depth due to the impact between the journal and bearing is evaluated as,

$\delta=e_{i j}-c$,

where $e_{i j}$ is the module of the eccentricity vector and $c$ is the radial clearance size. It should be noted that the radial clearance is a parameter specified by the user.

The candidate contact points on bodies $i$ and $j$ are $\mathrm{Q}_{i}$ and $\mathrm{Q}_{j}$, respectively. The position of these points in the journal and bearing are,

$\mathbf{r}_{k}^{\mathrm{Q}}=\mathbf{r}_{k}+\mathbf{A}_{k} \mathbf{s}_{k}^{\mathrm{Q}}+R_{k} \mathbf{n} \quad(k=i, j)$,

where $R_{i}$ and $R_{j}$ are the bearing and the journal radius, respectively.

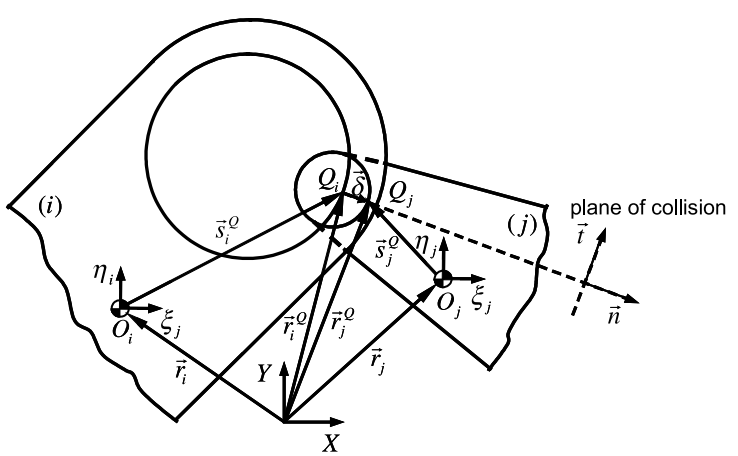

Fig. 5. Penetration depth between the journal and bearing during impact. 
The velocity of the contact points $\mathrm{Q}_{i}$ and $\mathrm{Q}_{j}$ in the global coordinate system is found by differentiating Eq. (14) with respect to time, yielding,

$\dot{\mathbf{r}}_{k}^{\mathrm{Q}}=\dot{\mathbf{r}}_{k}+\dot{\mathbf{A}}_{k} \mathbf{s}_{k}^{\mathrm{Q}}+R_{k} \dot{\mathbf{n}} \quad(k=i, j)$,

where the dot denotes the derivative with respect to time.

The components of the relative velocity of contact points in the normal and tangential plane of collision, shown in Fig. 6, are represented by $v_{\mathrm{N}}$ and $v_{\mathrm{T}}$, respectively. The relative normal velocity determines whether the contact bodies are approaching or separating. The relative scalar velocities, normal and tangential to the plane of collision, are found by projecting the relative impact velocity onto the respective direction, yielding,

$v_{\mathrm{N}}=\left(\dot{\mathbf{r}}_{j}^{\mathrm{Q}}-\dot{\mathbf{r}}_{i}^{\mathrm{Q}}\right)^{\mathrm{T}} \mathbf{n}$,

$v_{\mathrm{T}}=\left(\dot{\mathbf{r}}_{j}^{\mathrm{Q}}-\dot{\mathbf{r}}_{i}^{\mathrm{Q}}\right)^{\mathrm{T}} \mathbf{t}$,

where the tangent vector $\mathbf{t}$ is obtained by rotating vector n by $90^{\circ}$ in the anti-clockwise direction.

The normal and tangential forces, $\mathbf{f}_{\mathrm{N}}$ and $\mathbf{f}_{\mathrm{T}}$, respectively, act at the contact points. These forces are evaluated using a contact force model, for instance the Hertz's law, and a friction force model, for example the Coulomb's law. Similarly to the velocity analysis, the normal vector from the plane of collision is used as working direction for the contact forces. The contributions of the impact forces to the generalized vector of forces, $\mathbf{g}$ in Eq. (8), are found by projecting the normal and tangential forces onto the $X$ and $Y$ directions. These forces that act on the contact points are transferred to the center of mass of bodies $i$ and $j, \mathrm{O}_{i}$ and $\mathrm{O}_{j}$, respectively. Based on Fig. 7, the forces and moments acting on the center of mass of body $i$ are given by

$\mathbf{f}_{i}=\mathbf{f}_{\mathrm{N}}+\mathbf{f}_{\mathrm{T}}$,

$\mathbf{m}_{i}=-\left(y_{i}^{\mathrm{Q}}-y_{i}\right) f_{i}^{x}+\left(x_{i}^{\mathrm{Q}}-x_{i}\right) f_{i}^{y}$.

The forces and moments corresponding to the body $j$ are written as,

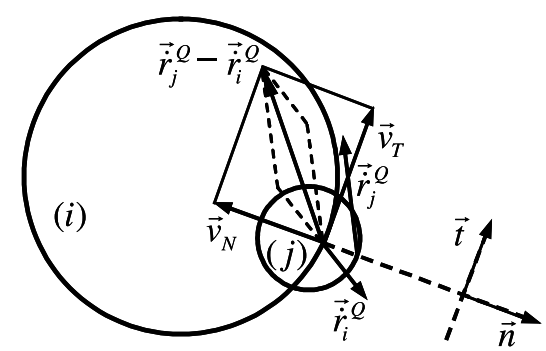

Fig. 6. Normal and tangential contact velocities.

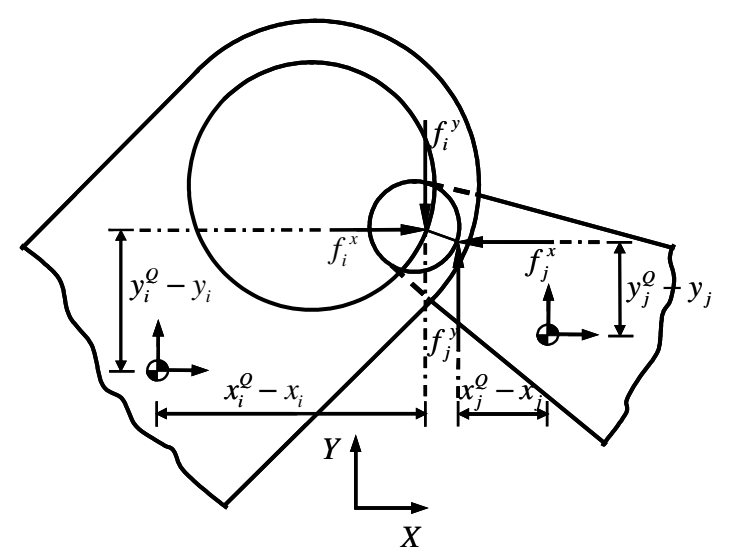

Fig. 7. Contact forces defined at the points of contact between journal and bearing.

$\mathbf{f}_{j}=-\mathbf{f}_{i}$,

$\mathbf{m}_{j}=\left(x_{j}^{\mathrm{Q}}-x_{j}\right) f_{j}^{y}-\left(y_{j}^{\mathrm{Q}}-y_{j}\right) f_{j}^{x}$.

The forces and moments given by Eqs. (18) through (21) are added to the generalized force vector $\mathbf{g}$ in Eq. (8). But before these quantities can be calculated it is necessary that the contact forces are evaluated using an appropriate contact model.

\section{Models for contact forces}

The contact force model used to evaluate the impact forces between the bearing and the journal plays a crucial role in the dynamic simulation of system which experiences impacts. The contact model must include information on the impact velocity, physical material properties and the geometric characteristics of the contacting bodies. Furthermore, the contact force model should also contribute to the stable integration of the system equations of motion. These characteristics are ensured by using a continuous contact force model in which the force and penetration vary in a continuous manner and for which some energy dissipation is included. In dynamic analysis, the deformation is known at every time step from the configuration of the system and the forces are evaluated based on the state variables. With the variation of the contact force during the contact period, the dynamic system response is obtained by simply including updated forces into the equations of motion. Since the equations of motion are integrated over the period of contact, this approach results in a rather accurate response. Furthermore, this methodology accounts for the changes in the system configuration during the contact period. 
The simplest contact model that is a candidate to be used in this work is the Kelvin-Voigt visco-elastic model, in which a spring-damper element between the contact bodies that represents the impact conditions. The spring represents the elasticity of the contacting bodies and the damper describes the energy dissipation during the impact process [24]. In this model, both the spring and damper are considered to be linear. During the unloading phase, the contact force is affected by the restitution coefficient. The linear Kelvin-Voigt contact force model is given as [6],

$\begin{cases}F_{\mathrm{N}}=K \delta & v_{\mathrm{N}}>0 \\ F_{\mathrm{N}}=e K \delta & v_{\mathrm{N}}<0\end{cases}$

where $K$ is the spring constant, $\delta$ is the relative penetration between the contacting bodies, $e$ is the restitution coefficient, and $v_{\mathrm{N}}$ is the normal relative velocity. The main difficulty with this model deals with the quantification of the spring constant, which depends on the geometry and physical properties of the contacting bodies. To consider the contact force as a linear function of penetration is not a good approximation as the nonlinear nature of the impact phenomenon suggests a more complex relation for contact force.

The best well-known impact force model between two isotropic spheres is the pure Hertz contact law, which is based on the elasticity theory [25]. The Hertz contact law relates the contact forces as a nonlinear power function of penetration, and can be expressed as,

$F_{\mathrm{N}}=K \delta^{n}$,

where $K$ is the generalized stiffness constant and $\delta$ is the relative penetration. The exponent $n$ is equal 1.5 for metals. The stiffness constant depends on the material properties and the geometric characteristics of the colliding bodies. For two spherical contacting surfaces the parameter $K$ is expressed as [26],

$K=\frac{4}{3 \pi\left(h_{i}+h_{j}\right)}\left(\frac{R_{i} R_{j}}{R_{i}-R_{j}}\right)^{\frac{1}{2}}$,

where the parameters $h_{i}$ and $h_{j}$ are given by

$h_{k}=\frac{1-v_{k}^{2}}{\pi E_{k}} \quad(k=i, j)$

being $v_{k}$ and $E_{k}$ the Poisson's coefficient and Young's modulus, respectively. Notice that the radius of curvature is defined positive for convex surfaces and negative for concave surfaces.

The Hertz contact law is a purely elastic contact force model, that is, it does not account for the energy dissipation during the impact. Compared to the KelvinVoigt, the advantages of the Hertz model are its nonlinearity, which represents well the compression phase of the contact between colliding bodies, and the physical characterization of the parameter $K$.

Based on Hertz theory, Dubowsky and Freudenstein [3] presented an expression, which relates the deformation and the impact force for a pin inside a cylindrical, as follows,

$\delta=F_{\mathrm{N}}\left(\frac{h_{i}+h_{j}}{L}\right)\left[\ln \left(\frac{L^{m}\left(R_{i}-R_{j}\right)}{F_{\mathrm{N}} R_{i} R_{j}\left(h_{i}+h_{j}\right)}\right)+1\right]$,

where $R_{i, j}$ and $h_{i, j}$ are the same parameters given by Eqs. (24) and (25), respectively, $L$ is the cylinder length, and the exponent $m$ is equal to 3. Since Eq. (26) is a nonlinear and implicit function for impact force, it is necessary to use an iterative technique, such as the Newton-Raphson method, to solve it for $F_{\mathrm{N}}$. Goldsmith [26] suggested a similar expression to Eq. (26), but with the exponent $m$ equal to 1 .

The ESDU-78035 Tribology Series [29] presented some expressions for impact forces analysis, for instance, for cylindrical contacts the proposed expression that is written as,

$\delta=F_{\mathrm{N}}\left(\frac{h_{i}+h_{j}}{L}\right)\left[\ln \left(\frac{4 L\left(R_{i}-R_{j}\right)}{F_{\mathrm{N}}\left(h_{i}+h_{j}\right)}\right)+1\right]$

in which the parameters are the same as in Eq. (26).

Hunt and Crossley [27] presented a nonlinear viscoelastic model to represent the energy transfer during the impact process. Based on the Hunt and Crossley work, Lankarani and Nikravesh [16] proposed a continuous contact force model in which a damping hysteretic factor is incorporated in order to account for the energy dissipation. This contact force model is expressed as,

$F_{\mathrm{N}}=K \delta^{n}\left(1+\frac{3\left(1-e^{2}\right)}{4} \frac{\dot{\delta}}{\dot{\delta}^{(-)}}\right)$,

where $K$ is the generalized stiffness constant expressed by Eq. (24) for the case of colliding spheres, $\delta$ is the relative penetration, $\dot{\delta}$ is the relative penetration velocity, and $\dot{\delta}^{(-)}$is the impact velocity. The restitution coefficient, $e$, reflects the type of impact, that is, for a perfectly plastic contact $e$ is null, and for a perfectly elastic contact $e$ is equal to 1. The use of Eq. (28) is limited by Love's criterion, that is, it is only valid for impact velocities lower than the propagation velocity of elastic waves across the solids [28].

The force-deformation diagrams for both spherical and cylindrical impact force models presented by Eqs. (22), (23), (26), (27) and (28) are displayed in Fig. 8. It is noticeable that the spherical and cylindrical forcedeformation diagrams are reasonably close. The model expressed by Eq. (28) is largely employed for mechanical impacts owning to its simplicity and easiness to implement in a computational program [6,15]. Further, this is the only contact model that accounts for the energy 


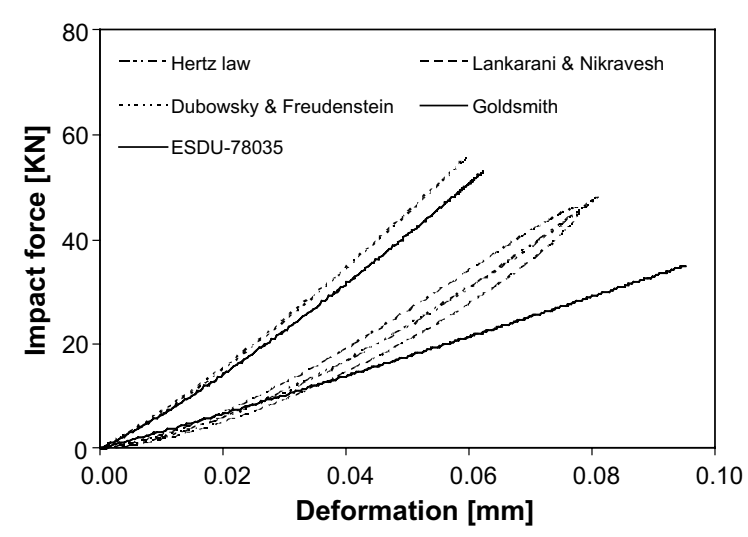

Fig. 8. Force-deformation diagrams for spherical and cylindrical contact force models.

dissipation during the impact process. The impact force models for cylindrical contact surfaces presented are purely elastic in so far as they do not account for the energy dissipation. Moreover, these expressions are nonlinear and implicit functions, which require the use of numerical iterative methods to obtain the impact force. This is a time consuming procedure that requires an approximate computer implementation to eliminate the iterative process.

\section{Demonstrative application to a slider-crank mechanism}

The slider-crank mechanism is chosen here to demonstrate the application of the methodologies presented in this work. The mechanism under consideration is made of four rigid bodies, two ideal revolute joints, one perfect translational joint and one revolute clearance joint that connects the slider and the connecting rod, as depicted by Fig. 9. The geometric and inertia data of the slider-crank mechanism is listed in Table 1.

The crank, which is the driving link, rotates with a constant angular velocity of $5000 \mathrm{rpm}$. The initial configuration of the mechanism is taken with the crank and the connecting rod collinear, and the journal and bearing centers coincident. Further, the initial conditions necessary to start the dynamic analysis are obtained

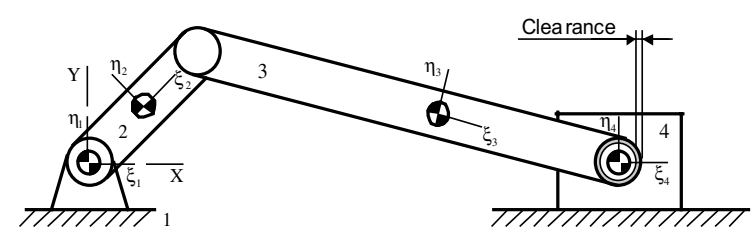

Fig. 9. Slider-crank mechanism with a revolute clearance.
Table 1

Governing properties for the slider-crank mechanism

\begin{tabular}{llll}
\hline $\begin{array}{l}\text { Body } \\
\text { Nr. }\end{array}$ & $\begin{array}{l}\text { Length } \\
{[\mathrm{m}]}\end{array}$ & $\begin{array}{l}\text { Mass } \\
{[\mathrm{kg}]}\end{array}$ & $\begin{array}{l}\text { Moment of inertia } \\
{\left[\mathrm{kg} \mathrm{m}^{2}\right]}\end{array}$ \\
\hline 2 & 0.05 & 0.30 & 0.00001 \\
3 & 0.12 & 0.21 & 0.00025 \\
4 & - & 0.14 & - \\
\hline
\end{tabular}

Table 2

Parameters used in the dynamic simulation for the slider-crank mechanism

\begin{tabular}{|c|c|c|c|}
\hline Bearing radius & $10.0 \mathrm{~mm}$ & Baumgarte- $\alpha$ & 5 \\
\hline $\begin{array}{l}\text { Restitution } \\
\text { coefficient }\end{array}$ & 0.9 & Baumgarte- $\beta$ & 5 \\
\hline Young's modulus & $207 \mathrm{GPa}$ & $\begin{array}{l}\text { Integration } \\
\text { step size }\end{array}$ & $0.00001 \mathrm{~s}$ \\
\hline Poisson's ratio & 0.3 & $\begin{array}{l}\text { Integration } \\
\text { tolerance }\end{array}$ & 0.000001 \\
\hline
\end{tabular}

from kinematic simulation of the slider-crank mechanism in which all the joints are considered to be ideal. The parameters used for the different models that characterize the problem and for the numerical methods required to solve the system dynamics are listed in Table 2.

The dynamic response of the slider-crank mechanism is obtained and represented by the evolution of velocity and acceleration of the slider, and the moment acting on the crank, which is necessary to keep the constant angular speed. Additionally, the relative motion between journal and bearing centers is plotted. Coupled with the penetration depth and surface properties, Hertz contact law with hysteresis damping factor, given by Eq. (28), is used to evaluate the contact force between the journal and bearing. Figs. 10-12 show the results for the case in which the clearance size is equal to $0.5 \mathrm{~mm}$. The results are compared to those obtained for ideal joint, and the time interval used corresponds to two complete crank rotations after steady-state has been reached.

In Fig. 10 it is observed that the existence of joint clearance influences the slider velocity by leading to a staircase like shape for the velocity versus time response. The periods of constant velocity observed for the slider mean that the journal can freely move inside the bearing boundaries. The sudden changes in velocity are due to the impact between the journal and the bearing. When a smooth change in the velocity curve of the slider is observed it indicates that the journal and the bearing are in continuous contact. The slider acceleration is subjected to high peaks caused by impact forces that are propagated through the rigid bodies of the mechanism, as observed in Fig. 11. The same phenomena can be observed in the curve of crank moment represented by Fig. 12. As far as the path of the journal center relative to the 


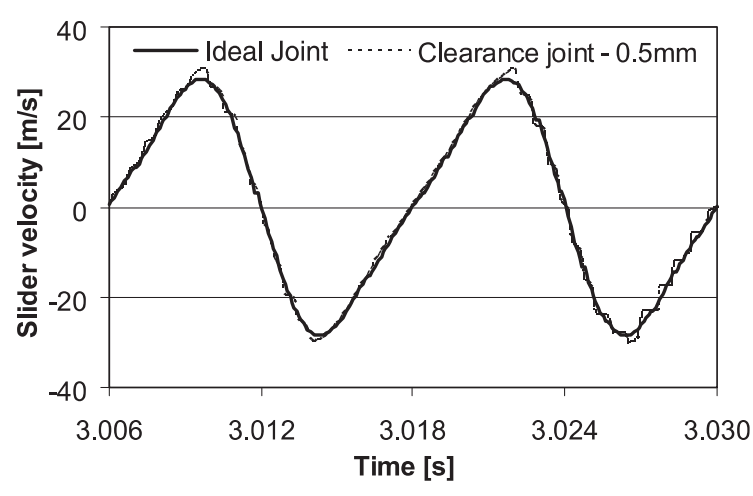

Fig. 10. Velocity of slider for the continuous contact force model given by Eq. (28).

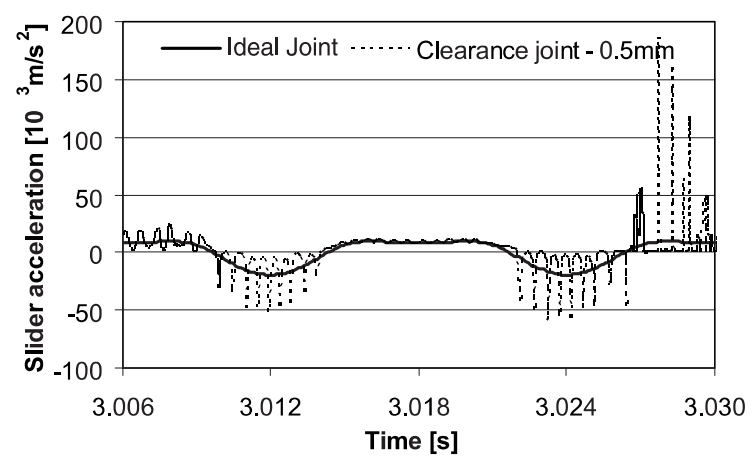

Fig. 11. Acceleration of slider for the continuous contact force model given by Eq. (28).

bearing center is concerned, different types of motion between the two bodies can be observed, namely, free flight, impact and rebound, and permanent or continuous contact. These types of motion are shown in Fig. 13(d).

The journal trajectories inside the bearing for two full crank rotations are used to illustrate the behavior of the slider-crank mechanism when different contact force models are used. The trajectories corresponding to each one of the models are pictured in Fig. 13. There it is observed that the contact models which do not include energy dissipation have long flight paths interrupted by short rebounds, which correspond to the contact between journal and bearing. The contact model by Lankarani and Nikravesh [16], which accounts for energy dissipation, presents long periods of contact between journal and bearing. In Fig. 13 the journal trajectories are presented by continuous lines that connect points. Each one of the points represent the position of the journal for a given time step. It can be observed that during the free flight the time step adopted by the integration algorithm is much larger that during the contact. When contact is de-

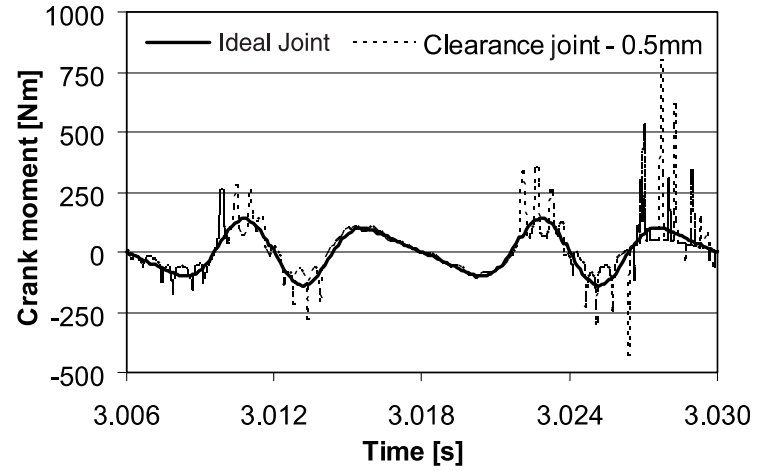

Fig. 12. Crank moment for the continuous contact force model given by Eq. (28).

tected, the integration time step decreases significantly, which show the importance of a varying time step integration algorithm for problems involving contact.

In addition to the journal trajectories, the crank moments for the different contact models and for the slider-crank with ideal joints are presented in Fig. 14. It is observed that all elastic contact models lead to the high peaks for the crank moments required to drive the crank with a constant angular velocity. The continuous contact force model proposed by Lankarani and Nikravesh presents much lower crank moment peaks, reflecting the dissipative energy features of the model. Such energy dissipation is also reflected by the long periods of time for which the crank moment is similar to that of the mechanism with ideal joints, observed in Fig. 14(e) and in Fig. 12, which is the same with a different scale.

\section{Concluding remarks}

A general methodology for dynamic characterization of mechanical systems with revolute clearance joints was presented in this work. The basic ingredients of the model proposed are the contact detection strategy and the contact force models used. The proposed procedures were demonstrated through the dynamical analysis of a slider-crank mechanism that has a revolute joint with clearance.

The Hertz contact theory based models are nonlinearity and do not account for the energy dissipation during the impact process. Therefore, the Hertz relation along with the modification to explain the energy dissipation in the form of internal damping can be adopted for modeling contact forces in a multibody system. The contact models for cylindrical contact areas do not present any advantage compared to the contact spherical models. Moreover, the cylindrical models are nonlinear and implicit functions, and the numerical iterative 

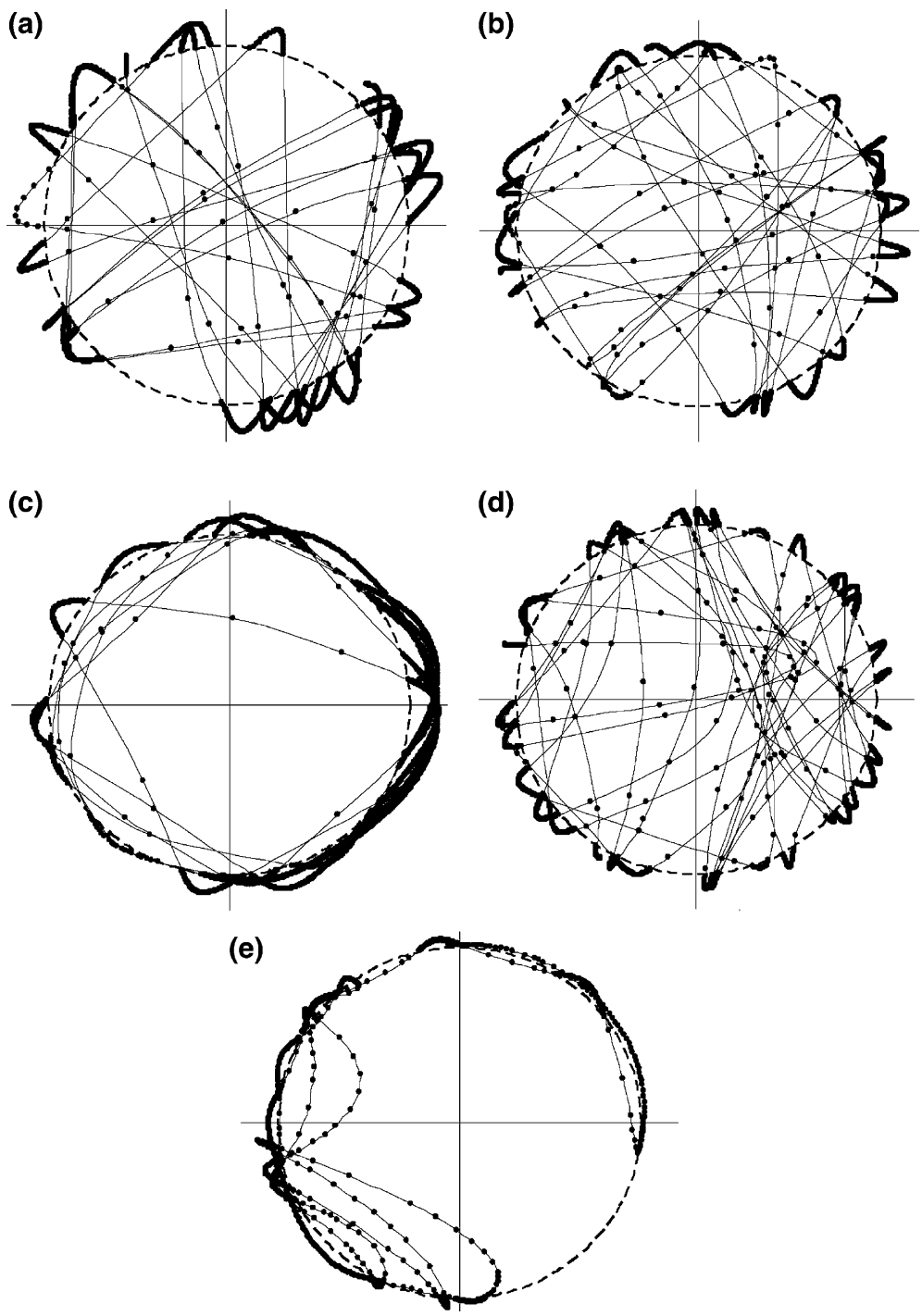

Fig. 13. Journal trajectory inside the bearing for the different contact models presented here. (a) Hertz contact law; (b) Dubowsky and Fraudenstein contact law; (c) Goldsmith contact model; (d) ESDU-78035 contact force model; (e) Lankarani and Nikravesh continuous contact force model.

procedure required to solve them is time consuming. These models are purely elastic in nature and cannot explain the energy dissipation during the impact process.

The different continuous contact force models that use an elastic contact theory lead to comparable results both in terms of journal trajectories and of crank moments. However, when energy dissipation is allowed to take place the peaks of the crank moments that are required to drive the mechanism with a constant angular speed are much lower than for the elastic models. This observation is consistent with the comparisons of the journal flights for the different models. It was observed that the energy dissipation of the continuous contact model proposed by Lankarani and Nikravesh results in long periods of time when the journal seats in the bearing, thus leading to a much smoother dynamic response of the system.

\section{Acknowledgements}

The support of the Fundação para a Ciência e a Tecnologia through the project POCTI/EME/2001/ 38281 entitled 'Dynamic of Mechanical Systems with Joint Clearances and Imperfections' is gratefully acknowledged. 

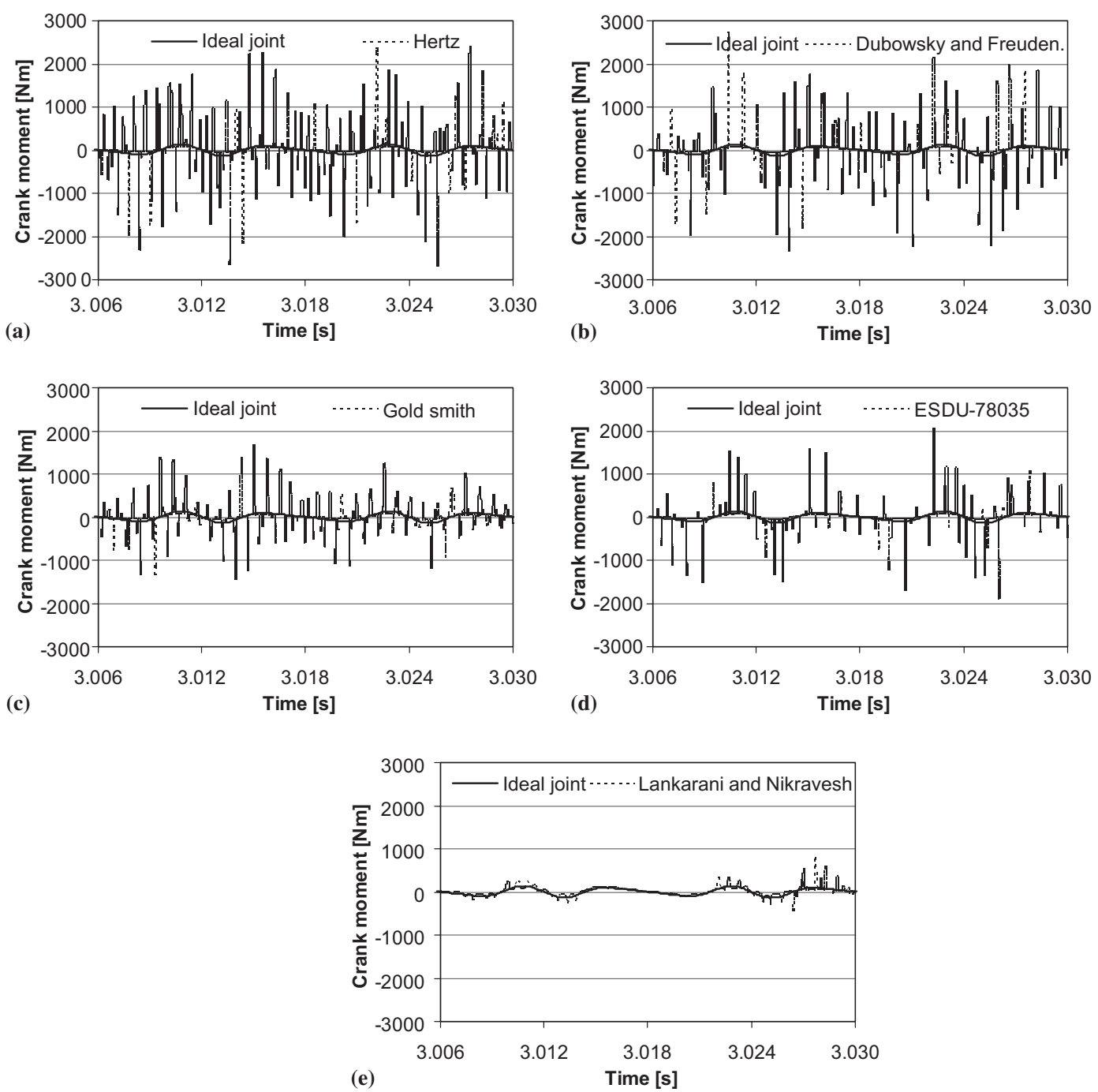

Fig. 14. Crank moment for the different contact models presented. (a) Hertz contact law; (b) Dubowsky and Fraudenstein contact law; (c) Goldsmith contact model; (d) ESDU-78035 contact force model; (e) Lankarani and Nikravesh continuous contact force model.

\section{References}

[1] Ryan RR. ADAMS - multibody system analysis software, multibody systems handbook. Berlin: Springer-Verlag; 1990.

[2] Smith RC, Haug EJ. DADS - dynamic analysis and design system, multibody systems handbook. Berlin: SpringerVerlag; 1990.

[3] Dubowsky S, Freudenstein F. Dynamic analysis of mechanical systems with clearances, Part 1: Formulation of dynamic model. J Eng Ind 1971;93(1):305-9.

[4] Dubowsky S, Freudenstein F. Dynamic analysis of mechanical systems with clearances, Part 2: Dynamic response. J Eng Ind 1971;93(1):310-6.

[5] Dubowsky S, Moening MF. An experimental and analytical study of impact forces in elastic mechanical systems with clearances. Mech Mach Theory 1978;13:451-65.
[6] Ravn P. A continuous analysis method for planar multibody systems with joint clearance. Multibody Syst Dyn 1998;2:1-24

[7] Bahgat BM, Osman MOM, Sankar TS. On the effect of bearing clearances in the dynamic analysis of planar mechanisms. J Mech Eng Sci 1979;21(6):42937.

[8] Bengisu MT, Hidayetoglu E, Akay A. A theoretical and experimental investigation of contact loss in the clearances of a four-bar mechanism. J Mech Transmissions Autom Des 1986;108:237-44.

[9] Dubowsky S, Gardner TN. Dynamic interactions of link elasticity and clearance connections in planar mechanical systems. J Eng Ind 1975;97(2):652-61.

[10] Claro JCP, Fernandes JPF. Influência da modelização das juntas na análise do desempenho de um mecanismo. 8as $\mathbf{J}$ Portuguesas Tribol, Aveiro 2002:215-9. 
[11] Wu CLS, Earles SWE. A determination of contact-loss at a bearing of a linkage mechanism. J Eng Ind 1977;99(2):37580.

[12] Haines RS. A theory of contact loss at resolute joints with clearance. J Mech Eng Sci 1980;22(3):129-36.

[13] Earles SWE, Seneviratne LD. Design guidelines for predicting contact loss in revolute joints of planar mechanisms. Proc Inst Mech Engrs, Part C 1990;204(1):9-18.

[14] Seneviratne LD, Earles SWE, Fenner DN. Analysis of a four-bar mechanism with a radially compliant clearance joint. Proc Inst Mech Engrs, Part C 1990;210(3):215-23.

[15] Schwab AL. Dynamics of flexible multibody systems: small vibrations superimposed on a general rigid body motion. $\mathrm{PhD}$ thesis, Delft University of Technology, The Netherlands, 2002.

[16] Lankarani HM, Nikravesh PE. A contact force model with hysteresis damping for impact analysis of multibody systems. J Mech Des 1990;112:369-76.

[17] Pereira MS, Nikravesh PE. Impact dynamics of multibody systems with frictional contact using joint coordinates and canonical equations of motion. Nonlinear Dyn 1996;9:5371.

[18] Pfeiffer F, Glocker C. Multibody dynamics with unilateral constraints. New York, NY: John Wiley and Sons; 1996.

[19] Ambrósio J. Rigid and flexible multibody dynamics tools for the simulation of systems subjected to contact and impact conditions. Eur J Solids A/Solids 2000;19:S23-44.
[20] Lankarani HM. Canonical equations of motion and estimation of parameters in the analysis of impact problems. PhD Dissertation, University of Arizona, Tucson, AZ, 1988.

[21] Nikravesh PE. Computer-aided analysis of mechanical systems. Englewood Cliffs, NJ: Prentice-Hall; 1988.

[22] Baumgarte J. Stabilization of constraints and integrals of motion in dynamical systems. Comput Methods Appl Mech Eng 1972;1:1-16.

[23] Shampine L, Gordon M. Computer solution of ordinary differential equations: the initial value problem. San Francisco: Freeman; 1975.

[24] Stronge WJ. Impact mechanics. Cambridge: Cambridge University Press; 2000.

[25] Timoshenko SP, Goodier JN. Theory of elasticity. New York: McGraw-Hill; 1970.

[26] Goldsmith W. Impact: the theory and physical behaviour of colliding solids. London: Edward Arnold Ltd; 1960.

[27] Hunt KH, Crossley FRE. Coefficient of restitution interpreted as damping in vibroimpact. J Appl Mech $1975 ; 7: 440-5$.

[28] Love AEH. A treatise on the mathematical theory of elasticity. 4th ed. New York: Dover Publications; 1944.

[29] ESDU-78035 Tribology Series. Contact phenomena I: stresses, deflections and contact dimensions for normallyloaded unlubricated elastic components. London: Engineering Sciences Data Unit; 1978. 